$\mathbb{T}$ periodica polytechnica

Mechanical Engineering

51/2 (2007) 59-62

doi: 10.3311/pp.me.2007-2.03

web: http://www.pp.bme.hu/me

(C) Periodica Polytechnica 2007

RESEARCH ARTICLE

\section{Life expectancy calculation of transient chaos in the 2D micro-chaos map}

\author{
Gábor Csernák / Gábor Stépán
}

Received 2008-01-18

\begin{abstract}
We analyse a simple model of a digitally controlled mechanical system, which may perform chaotic vibrations. As a consequence of the digital effects, i.e., the sampling and the round-off error, the behaviour of this system can be described by the socalled micro-chaos map. If dry friction is present in the system, it can stop the motion. In such cases the resulting behaviour is referred to as transient chaos, the duration of which can be closely related to the control time. We developed a method for the exact calculation of the mean lifetime $N_{m}$ of transient chaos in case of the 1D micro-chaos map, and showed that in certain cases $N_{m}$ characterizes the duration of chaotic transients better than the so-called escape rate. In the present paper, we try to extend these results to a $2 D$ version of the micro-chaos map.
\end{abstract}

\section{Keywords}

digital control $\cdot$ micro-chaos $\cdot$ two-dimensional maps

\section{Acknowledgement}

This research was supported by the Hungarian National Science Foundation under grant no. OTKA F049242, and by the János Bolyai Research Scholarship of the Hungarian Academy of Sciences.

\section{Gábor Csernák}

HAS-BUTE Research Group on Dynamics of Machines and Vehicles, H-1111 Budapest, Múegyetem rkp. 5., Hungary

e-mail: csernak@mm.bme.hu

\section{Gábor Stépán}

Department of Applied Mechanics, BME, H-1111 Budapest, Múegyetem rkp. 5., Hungary

\section{Mechanical Model}

Computers are widely applied to control the motions of machines [1]. Unfortunately, engineers are usually not aware of the fact that the stability properties of digital and analogue control systems are different. Digitally controlled systems often behave chaotically with very small vibration amplitudes. This phenomenon is often referred to as micro-chaos [2,3]. The sources of irregularity are the digital effects: the sampling, the round-off error and the processing delay. Taking into account these effects, the motion of the system can be described by a piecewise linear map, the micro-chaos map. If dry friction is present in the system, it can stop the motion in finite time, i.e., in these cases the resulting behaviour is transient chaotic [4].

In the present contribution, we analyse a simple model of a digitally controlled mechanical system which may perform transient chaotic vibrations. The motion of this system can be described by a 2D piecewise linear map. Our goal is to determine the mean lifetime of chaotic transients. For this purpose, we try to extend our earlier results [5-7], developed for the 1D microchaos map.

The mechanical model of a digitally controlled polishing tool is shown in Fig. 1. The model consists of a revolving cylinder (the tool), moving along a block (the workpiece). The shaft of the cylinder moves ahead with speed $v$, while its circumferential velocity is $v_{0}$. The characteristic $\mu\left(v_{\text {rel }}\right)$ of the friction force between the revolving polishing tool and the fixed workpiece is a mixture of the dry and viscous friction characteristics. At low relative speeds $v_{\text {rel }}=v_{0}-v$, this friction force is locally decreasing as $v_{\text {rel }}$ increases. Consequently, to stabilize the tool in a certain position, control force must be applied: the shaft of the polishing tool is driven by a DC motor, which exerts a control force $Q$, governed by a digital control system.

There is another friction force present in the system, denoted by $C$ in Fig. 1. This dry friction force may lead to the sudden disappearance of chaotic oscillations.

Note, that in real technological situations the polishing pressure - and consequently, the friction force between the tool and the workpiece - may vary in time. This effect may provide an additional irregular excitation to the system. Thus, the evolving 
motion can be chaotic in large scales as well. In engineering practice, special control systems are applied to hold the polishing pressure at a constant value [8]. This is why we will not take its variation into account.

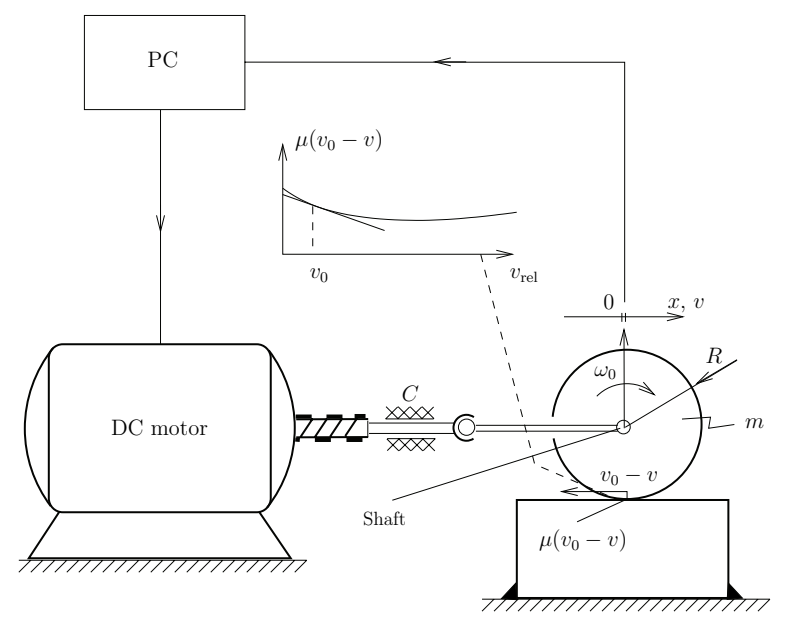

Fig. 1. Mechanical model of the polishing tool

The linearized, first order equation of motion of the system assumes the following form:

$m \dot{v}+m g \mu^{\prime}\left(v_{0}\right) v=m g \mu\left(v_{0}\right)-C \operatorname{sgn}(v) \underbrace{-m g \mu\left(v_{0}\right)-D v-P x}_{Q: \text { control force }}$,

where $m$ is the mass of the tool, while $D$ and $P$ denote the differential and proportional gains, respectively. For the sake of simplicity, we will denote $\left|\mu^{\prime}\left(v_{0}\right)\right|=-\mu^{\prime}\left(v_{0}\right)>0$ by $f$ in the following.

\section{Micro-chaos Map}

The computer samples the position $x$ and velocity $v$ at discrete time instances, $x_{j} \equiv x\left(t_{j}\right) \equiv x(j \tau), v_{j} \equiv v\left(t_{j}\right) \equiv v(j \tau)$, $j \in\{1,2, \ldots\} ; t_{j}=j \tau$ is the $j^{\text {th }}$ sampling instant, $\tau$ is the sampling time. Since some time is needed to process the measured signal, the force is exerted by the motor a bit after the sampling instant. This processing delay is often equal to the sampling time - we also consider this case. As a consequence of the delay, the control force exerted at $t_{j}$ depends on the data sampled at $t_{j-1}$. Moreover, due to the round-off error, the output signal must be an integer multiple of a finite resolution $h$.

If we give up the idea of stabilizing the shaft of the cylinder at a certain position $(P=0)$, we obtain the following 2D map, which is valid between two succesive sampling instants (for details see [5]):

$$
v_{j+1}=v_{j} e^{f g \tau}+\frac{1-e^{f g \tau}}{f m g}\left(h \operatorname{Int} \frac{D v_{j-1}}{h}+C \operatorname{sgn}\left(v_{j}\right)\right) .
$$

By introducing the new variable $y_{j}=D v_{j} / h$, and notations

$$
\begin{aligned}
a & =e^{f g \tau}>0 \\
b & =\left(e^{f g \tau}-1\right) \frac{D}{f m g}>0, \quad \text { and } \\
S & =\left(e^{f g \tau}-1\right) \frac{C D}{f m g h}>0,
\end{aligned}
$$

we obtain

$y_{j+1}=\left\{\begin{array}{cc}0 & \text { if }\left|a y_{j}-b \operatorname{Int}\left(y_{j-1}\right)\right| \leq S, \text { and } \\ a y_{j}-b \operatorname{Int}\left(y_{j-1}\right)-S \operatorname{sgn}\left(y_{j}\right) & \text { otherwise. }\end{array}\right.$

This map is a $2 \mathrm{D}$ version of the micro-chaos map, where the two variables are $y_{j-1}$ and $y_{j}$.

If we neglect the processing delay, we arrive at the following 1D map (see Fig. 2):

$y_{j+1}=\left\{\begin{array}{cl}a y_{j}-b \operatorname{Int}\left(y_{j}\right)-S & \text { if } S / a<y_{j}, \\ 0 & \text { if }-S / a \leq y_{j} \leq S / a, \text { and } \\ a y_{j}-b \operatorname{Int}\left(y_{j}\right)+S & \text { if } y_{j}<-S / a,\end{array}\right.$

This map is chaotic as it was proved in [3]. Since there is an at-

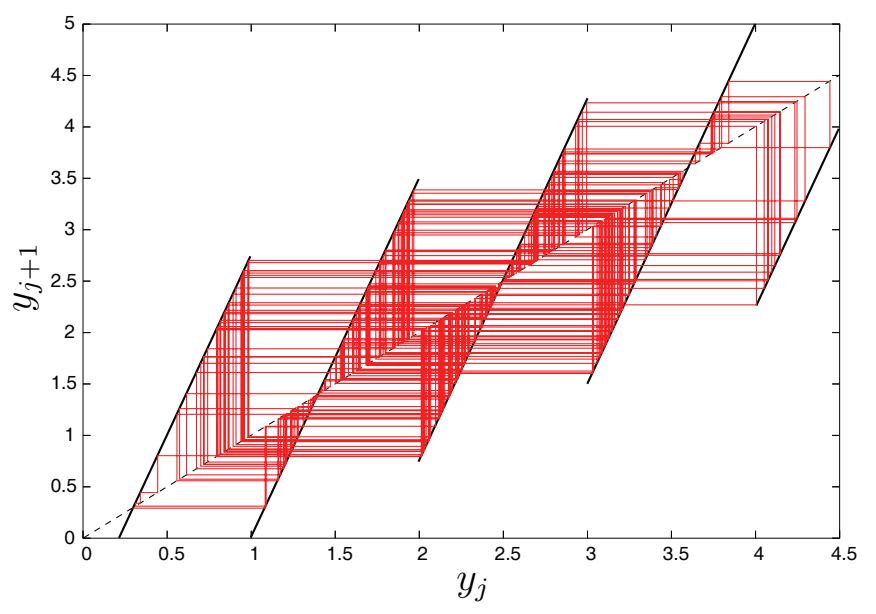

Fig. 2. 1D micro-chaos map, $a=3.5, b=2.75, S=0.75$

tracting domain $[-S / a, S / a]$ in the domain of definition of map (7), transient chaotic behaviour may occur. This attracting domain corresponds to the static dry friction $C$ that stops the shaft at low velocities. We developed a method for the exact calculation of the mean lifetime $N_{m}$ of transient chaos in case of this piecewise linear map [5]. If the fractal dimension of the chaotic repeller can be estimated easily, the results can be used for the quick estimation of the mean lifetime of chaotic transients $[6,7]$.

\section{Determination of the Lifetime of Transient Chaos}

We try to extend our results to the $2 \mathrm{D}$ version of the microchaos map (6), where the processing delay is not negligible, but equal to the sampling time. It was shown in [9], that the 2D map is also chaotic at $S=0$, i.e., in absence of dry friction. At certain parameters, the resulting solutions are transient chaotic, i.e., $y_{j}$ - which corresponds to the velocity - becomes zero after irregular oscillations.

As computer experiments showed, the repeller of the system - the set, within which the solutions wander before escaping to an attracting domain - is equivalent to a multi-valued version of the 1D map, since both consist of parallel line sections of slope $a$-see Figs. 2 and 3. The domain of definition of the 2D map is divided into parallel bands, according to the integer part of 


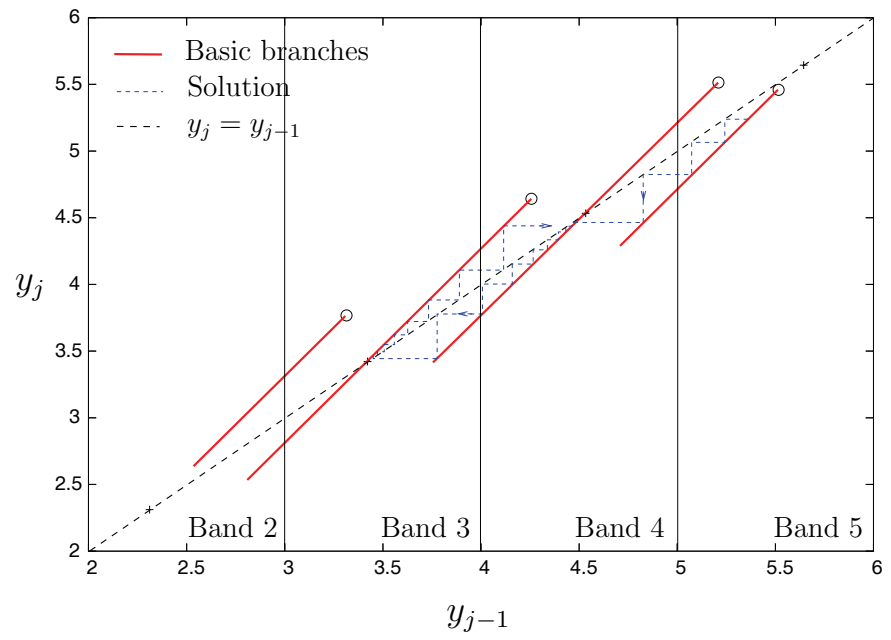

Fig. 3. Repeller of the 2D micro-chaos map, $a=1.45, b=0.5, S=0.04$

$y_{j-1}$. The successive iteration steps can be followed easily on a modified cob-web diagram. Starting at a certain initial point, the next point is found as follows: one projects the point "horizontally" to the diagonal, then "vertically" to the line of the repeller. In the multi-valued domains the appropriate branch is selected according to the previous value of the coordinate $y_{j-1}$.

To get a deeper insight into the mechanism of transient chaotic behaviour, we started our calculations with a simple case, with only one positive fixed point: $a=1.3, b=0.6$, and $S=0.156$. In this case the repeller can be enclosed in a square of size $a^{2}-S(1+a) \times a^{2}-S(1+a)$. This square is shaded in Figs. 4-6. The first few pre-images of the direct domain of attraction are presented in Fig. 4, the corresponding numbers showing the number of steps necessary to escape. The weighted mean of these numbers, where the weights are the areas of the rectangles, gives the mean lifetime of transient chaos. For example, in band 0 , there is a rectangle of size $1 \times S / a$,

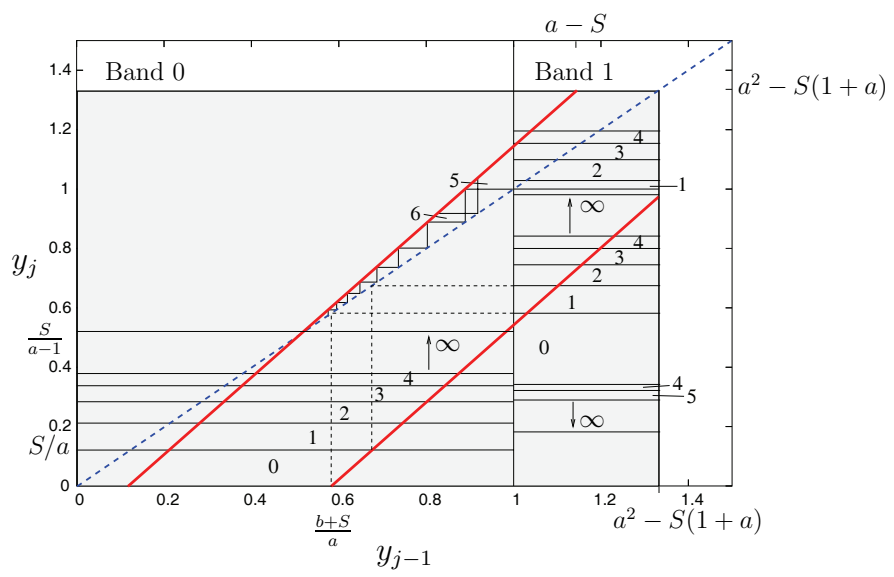

Fig. 4. Number of iteration steps, necessary to escape, $a=1.3, b=0.6$, $S=0.156$ (Algorithm of the calculation)

which belongs to the direct domain of attraction, i.e., its escape number equals 0 . The image of this rectangle is a neighbouring rectangle of size $1 \times S / a^{2}$ and escape number 1 . In this way a sequence of rectangles can be found. Summing up the heights of these rectangles one obtains the coordinate of the fixed point:

$$
\sum_{i=1}^{\infty} \frac{S}{a^{i}}=\frac{S}{a-1}
$$

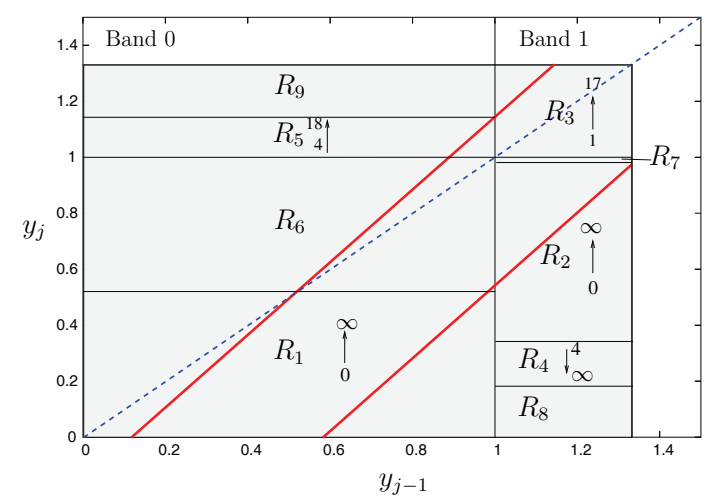

Fig. 5. Partition of the domain of definition at $a=1.3, b=0.6, S=0.156$

As it is shown in Fig. 5, the domain covered by this series of small rectangles is denoted by $R_{1}$. The width of $R_{1}$ is $W_{1}=1$, while the lower and upper border lines of this rectangle are the line $y_{j}=0$ and the line $y_{j}=S /(a-1)=0.52-$ this latter line crosses the fixed point.

Here the weighted sum of the escape numbers can be calculated quite easily:

$$
K_{1}=W_{1} \sum_{i=1}^{\infty}(i-1) \frac{S}{a^{i}}=1.73 \dot{3} .
$$

The domain covered by the repeller was divided into additional domains $R_{2} \ldots R_{9}$, where the corresponding weighted sums can be determined.

$\mathbf{R}_{2}$ : The width of this domain is $W_{2}=a^{2}-S(1+a)-1=$ 0.3312 . There is a large rectangle here with zero escape number at $y_{j} \in((b-S) / a,(b+S) / a)$. The rectangle with escape number 1 is the pre-image of another direct domain of attraction in band 0 (see Fig. 4). Thus, the weighted sum of the escape numbers can be determined just as in the case of $R_{1}$ :

$$
K_{2}=W_{2} \sum_{i=1}^{\infty}(i-1) \frac{S}{a^{i}}=0.57408
$$

$\mathbf{R}_{3}$ : The points in this domain are mapped into $R_{2}$ in one step. $W_{3}=W_{2}, y_{j} \in\left(1, a^{2}-S(1+a)\right)$. Since the point $(1,1)$ is mapped into the largest rectangle of $R_{2}$ with zero escape number (see Fig. 4), the first rectangle at the bottom of $R_{3}$ is mangled with height $h_{1}=(b+S)(a+1) / a^{2}-1$ and escape number 1 . Moreover, there is not enough area in this domain for an infinite sequence of rectangles with decreasing heights. Thus, there is another mangled rectangle at the top of $R_{3}$ with escape number 17 and height $h_{2}=a^{2}-S(1+a)-\left((b+S)(a+1) / a^{2}+\right.$ $\left.\sum_{i=3}^{17} S / a^{i}\right)$. Thus, the weighted sum of the escape numbers can be obtained as

$$
K_{3}=W_{3}\left(1 h_{1}+17 h_{2}+\sum_{i=3}^{17}(i-1) \frac{S}{a^{i}}\right) \approx 0.51621 .
$$


$\mathbf{R}_{4}$ : This domain is bordered by the lines $y_{j}=S /(a(1-a))+$ $(S+b) / a$ and $y_{j}=(b-S) / a$ and is mapped into another domain in the negative half-plane, which is similar to $R_{1}$. The upper border line of $R_{4}$ is mapped into a rectangle with escape number 3. Thus, there is a mangled rectangle at the top of $R_{4}$ with height $h_{3} \approx 0.001995$ and escape number 4 . Since $W_{4}=W_{2}$, the weighted sum of the escape numbers is

$$
K_{4}=W_{4}\left(4 h_{3}+\sum_{i=6}^{\infty}(i-1) \frac{S}{a^{i}}\right) \approx 0.412969 .
$$

$\mathbf{R}_{\mathbf{5}}$ : This domain is found at $y_{j} \in(1, a-S)$, and mapped into $R_{3}$ in one step, and there are mangled rectangles at its top and bottom, with escape numbers 4 and 18 , and heights $h_{5} \approx 0.008078$ and $h_{6}=h_{2} / a \approx 0.00049$, respectively. The weighted sum of the escape numbers, with $W_{5}=1$ :

$$
K_{5}=W_{5}\left(4 h_{5}+18 h_{6}+\sum_{i=6}^{18}(i-1) \frac{S}{a^{i}}\right) \approx 1.1096649
$$

$\mathbf{R}_{\mathbf{6}}$ : This domain is in $y_{j} \in(S /(a-1), 1)$ and consists of the images of $R_{5}$, thus, the weighted sum of the escape numbers can be obtained as an infinite sum of expressions like in $K_{5}$, with increasing escape numbers and geometrically decreasing rectangle sizes.

$$
\begin{aligned}
K_{6}= & W_{6}\left(\sum_{i=1}^{\infty}(4+i) \frac{h_{5}}{a^{i}}+\sum_{i=1}^{\infty}(18+i) \frac{h_{6}}{a^{i}}+\right. \\
& \left.\sum_{j=1}^{\infty} \sum_{i=6}^{18}(i-1+j) \frac{S}{a^{i+j}}\right) \approx 5.77888 .
\end{aligned}
$$

$\mathbf{R}_{7}$ : This domain is found between $R_{2}$ and $R_{3}$. The points of this domain are mapped into the bottom of $R_{6}$ in one step. Thus, the structure of these domains is similar, but $R_{7}$ consists of mangled sub-rectangles. After a straightforward but long calculation, we obtain the weighted sum of the escape numbers:

$$
K_{7} \approx 0.147603 \text {. }
$$

$\mathbf{R}_{\mathbf{8}}$ : This domain is in $y_{j} \in(0, S /(a(1-a))+(S+b) / a)$ and is mapped in two steps in a domain in the negative half-plane that corresponds to $R_{6}$. Due to the symmetry, we can calculate as if it would map into $R_{6}$, next to the fixed point. The calculation of $K_{8}$ is similarly long but easy as in the case of $K_{7}$.

$$
K_{8} \approx 0.935384
$$

R9: This domain is in $y_{j} \in\left(a-S, a^{2}-S(1+a)\right)$ and is mapped in two steps onto $R_{7}$ and into $R_{2}$ and $R_{3}$. The calculation is even longer than so far, but can be performed:

$$
K_{9} \approx 1.369039
$$

The mean escape number can be obtained as

$$
N_{m}=\frac{\sum_{i=1}^{9} K_{i}}{\left(a^{2}-S(1-a)\right)^{2}}=7.0973
$$

where the denominator is the area of the repeller (the shaded rectangle in Fig. 4).

We also performed numerical simulations and determined the number $N$ of steps necessary to escape from 600 points of the repeller of map (6). The initial values of $y_{j-1}$ were chosen to be 0,1 , and 2 , while the initial values of $y_{j}$ were uniformly distributed between 0 and 2. The obtained mean value is $N_{m}^{s i m}=$ $7.0669 \pm 0.0369$, which is equal to the calculated mean lifetime (18) within the standard error.

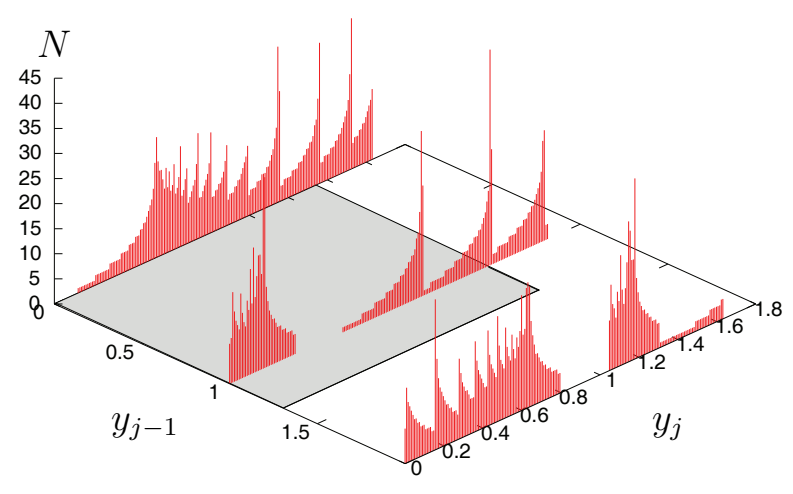

Fig. 6. Number of iteration steps, necessary to escape, $a=1.3, b=0.6$, $S=0.156$ (Numerical simulation)

\section{Conclusions}

A two-dimensional version of the micro-chaos map was introduced. Exploiting the similarity between the 1D micro-chaos map and the attractor of the 2D map, we started to extend the area of application of our methods for the estimation of the lifetime of transient chaos in case of two-dimensional maps. Our analitical results were checked by numerical simulation in a particular example.

\section{References}

1 Kuo B C, Digital Control Systems, SRL Publishing, Champaign, IL, USA, 1977.

2 Enikov E., Stépán G, Microchaotic Motion of Digitally Controlled Machines, Journal of Vibration and Control 4 (1998), 427-443.

3 Haller G, Stépán G, Micro-Chaos in Digital Control, J. Nonlinear Sci. 6 (1996), 415-448.

4 Tél T, Transient Chaos: Experimental Study and Characterization of Chaos (Hao Bai-lin, ed.), Vol. 3, World Scientific Publishing Company, Singapore, 1990.

5 Csernák G, Stépán G, Life Expectancy of Transient Microchaotic Behaviour, J. Nonlinear Sci. 15 (2005), no. 2, 63-91.

6 , Fractal Dimension as Measure of Control Time, Periodica Polytechnica 48 (2004), no. 1, 17-25.

7 , Quick Estimation of Escape Rate with the Help of Fractal Dimension, Communications in Nonlinear Science and Numerical Simulation 11 (2005), no. 5, 595-605.

8 Yi A.Y., Hezlep M., Pol T, A computer controlled optical pin polishing machine, Journal of Materials Processing Technology 146 (2004), no. 2, 156162.

9 Csernák G, Stépán G, Sometimes Digital Control Leads to Chaos, Proc. of the 14th International Congress on Sound and Vibration, 2007. 